\title{
LOCUCIONES E ILOCUCIONES: SEARLE Y AUSTIN*
}

\author{
EDUARDO A. RABOSSI \\ Consejo Nacional de \\ Investigaciones Científicas y Técnicas \\ de la República Argentina
}

\section{Comentario preliminar}

1. En un interesante artículo, "Austin on Locutionary and Illocutionary Acts", John Searle manifiesta que al investigar la noción de acto ilocucionario encontró inadecuada la noción de acto locucionario, tal como la emplea Austin, y que esa circunstancia lo llevó a adoptar una distinción diferente, a saber, la distinción entre acto locucionario y acto proposicional. En las secciones I y II de dicho artículo Searle expone "[sus] razones para rechazar la distinción trazada por Austin". El análisis que ofrece - sin duda atractivo- tiene como objeto principal formular y dar fundamento a dos críticas de peso: (a) que "la distinción locucionario-ilocucionario no es totalmente general, porque algunos actos locucionarios son actos ilocucionarios". (LIA, 408) y (b) que "todos los miembros de la clase de actos locucionarios (realizados al emitir oraciones completas) son miembros de la clase de actos ilocucionarios, porque todo acto rético, y en consecuencia todo acto locucionario, es un acto ilocucionario". (LIA, 413). En las secciones III y IV Searle expone sus porpios puntos de vista sobre

* Este artículo es una versión modificada del trabajo "Actos lingüísticos: locuciones e ilocuciones" que leí el lo. de junio pmo. pdo. ante la Sociedad Argentina de Análisis Filosófico y que discutió el Prof. Genaro Carrió. Agradezco a Carrió las críticas formuladas, pues me han permitido mejorar la presentación original. El artículo forma parte de una investigación sobre el tema: Actos lingüísticos y acción humana, iniciada en la Universidad de $\mathbf{0 x}$ ford, en 1970, como Guggenheim Fellow y continuada como investigador del Consejo Nacional de Investigaciones Científicas y Técnicas de la República Argentina.

1 Philosophical Review, 77 (1968), pp. 405-424. En adelante LIA. 
varios temas centrales de la teoría de los actos lingüísticos. Hacen aparición allí el denominado 'principio de expresibilidad" ("Todo lo que se puede tener intención de decir, se puede decir"). la referencia a la mayor o menor especifidad de las fuerzas ilocucionarias de las expresiones, la distinción entre actos ilocucionarios y actos proposicionales, los indicadores de fuerza ilocucionaria y los indicadores de contenido proposicional, etc. Quien haya leído Speech Acts $^{2}$ advertirá que las secciones III y IV abarcan temas y tesis centrales de los capítulos primero y segundo del libro; recordará, además, que al adoptar la terminología austiniana de 'acto ilocucionario' Searle remite al lector a "Austin on Locutionary and Illocutionary Acts" y que lo hace con la siguiente aclaración: "Empleo la expresión 'acto ilocucionario' con algunas dudas, dado que no acepto la distinción que traza Austin entre actos locucionarios e ilocucionarios"." por razones perfectamente comprensibles Searle omite exponer en Speech Acts los argumentos en los que fundamenta tal rechazo. Ellos se encuentran desarrollados, como he señalado, en las secciones I y II del artículo que nos ocupa.

2. En este trabajo me propongo analizar críticamente el planteo que hace Searle al fundamentar su rechazo de la ya clásica distinción trazada por Austin entre actos locucionarios y actos ilocucionarios. ${ }^{\star}$ No es mi intención, por cierto, criticar la estrategia general que subyace a tal planteo, pues considera correcto que el cuestionamiento de la distinción locucionario-ilocucionario se lleve a cabo -tal como pretende hacerlo Searle- dentro de un marco acotado, básicamente, por distinciones y refinamientos teóricos formulados por el propio Austin. Lo que me interesa cri-

2 John Searle, Speech Acts, Cambridge, Cambridge University Press, 1969.

3 John Searle, op. cit., p. 23, nota 1.

4 Cf. John L. Austin, How To Do Things with Words, Oxford, Clarendon Press, 1962. (Palabras y Acciones, Buenos Aires, Paidos, 1971, traducción de Genaro Carrió y Eduardo Rabossi.) En adelante PA. En las citas correspondientes, los números en cursivas corresponden a las páginas de la traducción al castellano. 
ticar, en cambio, es la trama argumentativa que desarrolla Searle, dado que en la misma se desliza una dudosa interpretación del enfoque de Austin $\mathrm{y}$, además, se introducen distinciones y se formulan aseveraciones que distan de ser claras, que no poseen un contenido preciso y que padecen, en general, del mismo grado de indeterminación que ostentan algunas piezas claves del enfoque austiniano. Todo esto mueve a pensar que las dos críticas que formula Searle no poseen el eficaz peso teórico que él les atribuye, que tales críticas pueden ser "absorbidas", en líneas generales, dentro del planteo de Austin y que, bien vistas las cosas, exhiben una vacuidad teórica que hace aconsejable que se las desestime por irrelevantes.

Es conveniente aclarar que el planteo crítico que me propongo desarrollar no implica, en modo alguno, ignorar o minimizar la legitimidad del problema de fondo que preocupa básicamente a Searle, a saber, dar cuenta de las dificultades teóricas que se plantean en torno a las nociones de acto locucionario y de acto ilocucionario, tal como han sido introducidas por Austin, y lograr, consiguientemente, un refinamiento conceptual adecuado. Que el propio Austin expresara reservas respecto del contenido que dio a esas nociones es un dato sintomático que autoriza a omitir mayores aclaraciones acerca del interés y de la relevancia de dicha investigación. ${ }^{5}$

Es también conveniente advertir que mis observaciones críticas no pretenden producir consecuencias apocalípticas. No creo, por ejemplo, que si llegara a probar la eventual falta de consistencia de las dos críticas formuladas por Searle, se derrumbaría inexorablemente el esquema teórico desarrollado en Speech Acts. El valor real de tal esquema es independiente de la pertinencia de las críticas que pue-

5 Cf.: "Hemos distinguido, pues, en forma gruesa tres tipos de actos: el locucionario, el ilocucionario y el perlocucionario... ${ }^{2}[$ "...(1) Todo esto no está claro (2) y en todos los sentidos relevantes ( (A) y (B) como cosas distintas a (C) ¿no serán todas las expresiones, expresiones realizativas?"]". (PA, p. 103, nota 1: p. 147, nota 2). Véase, además, PA, pp. 94-95, 139-139. 
dan hacerse a Austin. Lo que sí creo es que de probarse la inconsistencia de las críticas formuladas por Searle, se habrá probado que una de las posibles líneas argumentativas que llevan (o han llevado) a ese esquema, carece de asidero. $Y$ éste no es, por cierto, un punto mínimo. En rigor de verdad, es un punto que posee importancia para los que consideramos - igual que Searle- que en la doctrina de los actos lingüísticos sugerida por Austin se encuentra en germen un planteo novedoso y fructifero, pero que creemos - quizá en oposición a Searle- que todavía sigue en pie la tarea de proponer una formulación real mente adecuada de tal doctrina.

3. Las abundantes referencias que se harán a la doctrina de Austin, sea a través de los comentarios de Searle, sea a través de mis propios comentarios, impone detenernos en PA con el fin de hacer explícitos varios temas relevantes para nuestra discusión. Esta tarea -en lo posible breve y necesariamente sinóptica- nos ocupará en primer término $(\S \S 4$ y 5$)$. Expondré y criticaré luego la primera de las críticas formuladas por Searle $(\$ \S 6,7$ y 8$)$ y a continuación expondré y criticaré la segunda de sus críticas ( $\$ \S 9$, 10 y 11). A modo de conclusión, formularé algunos comentarios sobre los temas discutidos y sobre su relación con el enfoque teórico general de Searle ( $\$ 12$ ).

\section{Algunos aspectos de la doctrina de Austin}

4. Dentro del contexto de PA la doctrina de los actos lingüísticos hace aparición en la parte final de la Conferencia VII luego de haberse mostrado el fracaso - dramáticamente regulado por Austin - de los distintos criterios ofrecidos para distinguir los realizativos de los constatativos. Se trata, pues, de "comenzar de nuevo" y de llevar hasta sus últimas consecuencias teóricas la ecuación decir algo (es) hacer algo, ecuación que la noción de realizativo primero, y las discusiones en torno a la relación entre realalizativos y cons- 
tatativos, después, han colocado en primerísima línea. Sin embargo, este giro no implica que la distinción realizativoconstatativo desaparezca, pues "... está respecto de la doc-. trina de los actos locucionarios e ilocucionarios en el acto lingüístico total, en la posición de una teoría especial frente a una teoría general." (PA, 147, 195).

Es imposible, y a nuestros fines irrelevante, intentar ofrecer una sintesis acabada de dicha doctrina general. Pero un procedimiento a seguir, útil y quizá interesante, puede ser el siguiente: precisar varios temas generales que tengan relevancia para nuestra discusión y citar, bajo el rótulo correspondiente, los textos más representativos de PA. Los temas generales pueden ser los siguientes: (1) índole de la tarea a realizar, (2) resultado inmediato de la realización de tal tarea, (3) tema central de investigación, (4) consecuencias teóricas de la elección de dicho tema, (5) problemas teóricos específicos, a saber, (a) naturaleza de los actos y relaciones entre los actos, (b) criterios de identidad de los actos, (c) carácter convencional de los actos, (d) características de los enunciados que describen distintos tipos de actos y (e) significado y fuerza.

(1) Austin describe la indole de la tarea a realizar como la especificación de los distintos sentidos en que decir algo es hacer algo. $\mathrm{Y}$ desde el comienzo mismo se sugieren tres sentidos básicos: "Es menester que consideremos de un modo más general los sentidos en que decir algo puede ser hacer algo, o en que al decir algo hacemos algo (y también, quizá, considerar el caso diferente en el que por decir algo hacemos algo)". (PA, 91, 135-136; cf. también $94,138$ y 120,153$)$. Austin ofrece varias versiones alternativas de la índole de la tarea que se propone llevar a cabo, por ejemplo, “... afinar nuestra reflexión sobre las circunstancias que rodean el 'emitir una expresión'". (PA, $92,136), 0^{\prime \prime}$. distinguimos un grupo de cosas que hacemos al decir algo". (PA, 108, 153), o "...tenemos tres sentidos o dimensiones diferentes, si no más, de la expresión 'el 
uso de una oración' o 'el uso del lenguaje' (y, por cierto, también hay otras)". (PA, 108, 153), o "La importancia de estas investigaciones es obvia respecto de la pregunta general ‘¿cómo están relacionadas entre sí las diversas descripciones posibles de lo que hago?" (PA, 122, 169). Pero aunque estas versiones son diferentes entre sí, creo que puede afirmarse que ninguna de ellas modifica esencialmente la descripción formulada originariamente acerca de la índole de la tarea a realizar. Más bien, algunas son meras variantes de tal descripción y otras le son complementarias.

(2) El resultado inmediato de la realización de dicha tarea es la especificación de un grupo de sentidos distintos de la expresión 'decir algo' que permiten especificar, a su vez, diferentes actos que realizamos diciendo algo. El primer grupo de sentidos abarca 'decir algo' en el significado pleno y normal de 'decir': “. . . decir algo A.a) es siempre el acto de emitir ciertos sonidos (un acto fonético) ....A.b) es siempre el acto de emitir ciertos vocablos o palabras, esto es, sonidos de ciertos tipos pertenecientes a cierto vocabulario y en cuanto pertencientes a él, emitidos en una construcción [gramatical] determinada... Podemos llamar a este acto, un acto 'fático'...A.c), generalmente, es realizar el acto de usar [la expresión emitida en el acto fático] o sus part is constituyentes con un 'sentido' más o menos definido y una 'referencia' más o menos definida ('sentido' y 'referencia' que tomadas conjuntamente equivalen a 'significado'). Podemos llamar a este acto, un acto 'rético'". (PA, 92-93, 136-137). 'Llamo al acto de 'decir algo', en esta acepción plena y normal, realizar un acto locucionario". [(A)] (PA, 94, 138).

Ahora bien, “...cuando realizamos un acto locucionario, usamos el lenguaje, pero ¿en qué modo preciso lo estamos usando en determinada ocasión?... Es muy diferente que estemos aconsejando o meramente sugiriendo o realmente ordenando, o que estemos prometiendo en un sentido 
estricto o sólo anunciando una vaga intención, etc. Estas cuestiones las discutimos preguntando si ciertas palabras (una determinada locución) tenían la fuerza de una pregunta, o debian haber sido tomadas como una apreciación, etc. . . realizar un acto en este nuevo sentido es realizar un acto ilocucionario [(B) ]. Esto es, llevar a cabo un acto al decir algo, como cosa diferente de llevar a cabo el acto de decir algo". (PA, 99, 143-144).

Por fin, "...hay un tercer sentido (C), según el cual realizar un acto locucionario y, con él, un acto ilocucionario puede ser también realizar un acto de otro tipo. A menudo, e incluso normalmente, decir algo producirá ciertas consecuencias o efectos sobre los acontecimientos, pensamientos o acciones del auditorio, o de quien emite la expresión, o de otras personas... Llamaremos a la realización de un acto de este tipo, la realización de un acto perlocucionario". (PA, 101, 145).

(3) La identificación de estos tipos de actos vía la elucidación de los sentidos diferentes en que decir algo es hacer algo permite, entre otras cosas, dar ubicación al tema central de la investigación. Ese tema central lo constituyen los actos ilocucionarios. "En estas conferencias nuestro interés consiste especialmente en aprehender el acto ilocucionario y en contrastarlo con los otros dos". (PA, 103, 147). Aunque es bastante obvio que al realizar tal contraste Austin no observa un equilibrio total. Y la razón que da para justificar el "desequilibrio" en el que incurre es que "...la que parece crear más dificultades es la distinción entre ilocuciones y perlocuciones; de ella nos ocuparemos ahora y sólo tomaremos al pasar la distinción entre ilocuciones y locuciones". (PA, 109, 154). Esta valoración no es del todo correcta pues, de hecho, la relación que más dificultades ha creado es la que se da entre ilocuciones y locuciones. Pero - dicho sea de paso en favor de Austin - no cabe duda de que su juicio fue motivado fundamentalmente por el carácter novedoso de las relaciones entre ilocuciones y perlocu- 
ciones y, en consecuencia, por la necesidad de centrar el análisis en la dirección menos transitada por los teóricos del lenguaje.

(4) La elección de los actos ilocucionarios como tema central de investigación tiene importantes consecuencias teóricas, especialmente en lo que respecta a los fenómenos específicos a estudiar. Dicha elección está determinada, en gran parte, por el hecho de que "...desde hace años venimos advirtiendo cada vez con mayor claridad que la ocasión en que una expresión se emite tiene gran importancia, y que las palabras usadas tienen que ser 'explicadas', en alguna medida, por el contexto dentro del cual se intenta usarlas o fueron usadas en un intercambio lingüístico." (PA, 100, 144). De esto se extrae que "... lo que tenemos que estudiar no es la oración sino el acto de emitir una expresión en una situación linguística". (PA, 133, 185). En términos más genrales, "...el acto lingüístico total en la situación lingüística total, constituye el fenómeno real que, en última instancia, estamos tratando de elucidar". (PA, 147, 196).

(5) Los problemas teóricos que surgen en relación con el planteo de Austin son muchos y de índole variada. Algunos de tales problemas, que tendrán peculiar relevancia en la discusión que sigue, son estos:

(a) ¿Cuál es la naturaleza de los actos a los que se refiere Austin y cuáles son las relaciones que se dan, o que pueden darse, entre dichos actos? Al respecto, Austin ofrece varias pistas interesantes. ". . [ [h] ablando en términos generales, el acto locucionario, en igual medida que el acto ilocucionario, sólo es una abstracción: todo acto genuino es ambas cosas a la vez. (Esto es similar a lo que ocurre con el acto 'fático', el acto 'rético', etc., que también son abstracciones). Pero, por supuesto, típicamente dstinguimos entre los diferentes 'actos' abstraídos por medio de los lapsos posibles. Es decir, por medio de los diferentes tipos de sinsentidos que pueden producirse al llevar a cabo tales 'actos'". (PA, 146, 194). Y en relación con este punto resulta 
interesante citar el siguiente párrafo: "El pheme [la expresión emitida en el acto fático] es una unidad del lenguaje; su deficiencia típica es ser un sinsentido- carecer de significado. Pero el rheme [la expresión emitida en el acto rético] es una unidad del habla (speech); su deficiencia típica es ser vago, o vacuo, u obscuro, etc." (PA, 98, 142). A su vez, en concordancia con la observación de que todo acto lingüístico genuino es a la vez un acto locucionario y un acto ilocucionario, Austin señala que "... realizar un acto locucionario es, en general, eo ipso, realizar un acto ilocucionario". (PA, 98, 143). Además, "...para realizar un acto 'fático' tengo que realizar un acto 'fonético', o, si se prefiere, al realizar uno estoy realizando el otro. (Esto no significa, sin embargo, que los actos 'fáticos' sean una subclase de los fonéticos, esto es, que pertenezcan a la clase de estos últimos)". (PA, 96, 139-140). Y ante la eventual objeción de que la noción de acto no posee la claridad adecuada - una objeción correcta- Austin remite a la construcción de una teoría general de la acción (cf. PA, 106, 150-151).

(b) ¿Cuáles son los criterios de identidad de los actos en cuestión? Austin es muy parco al respecto: “Aquí no nos interesan mucho cuestiones como la de saber cuándo un pheme o un rheme es el mismo, sea en el sentido del 'tipo' (type) o del 'caso concreto' (token), ni si hay uno o más phemes o rhemes, etc. pero, por supuesto, es importante recordar que el mismo pheme (caso concreto del mismo tipo) puede ser usado en distintas ocasiones de expresión con un sentido y una referencia diferentes, y ser así un rheme diferente. Cuando se usan phemes distintos con el mismo sentido y referencia, podríamos hablar de actos 'réticamente' equivalentes (en cierto sentido, 'el mismo enunciado') pero no del mismo rheme o de los mismos actos 'réticos' (que son el mismo enunciado en otro sentido que importa el uso de las mismas palabras)". (PA, 97-98, 141-142). 
(c) ¿Qué carácter poseen los tipos de actos de los que habla Austin? ¿Son todos ellos actos convencionales, por ejemplo? Austin admite que tanto los actos locucionarios como los actos ilocucionarios tienen carácter convencional, no así los perlocucionarios. No es explícito, por cierto, acerca de qué entiende por 'convención', especialmente cuando se aplica a actos ilocucionarios. En un párrafo bastante críptico señala lo siguiente: "Hablar del 'uso del "lenguaje" para prometer o advertir', parece exactamente igual a hablar del 'uso del "lenguaje" para persuadir, excitar, alarmar, etc.'. Sin embargo, el primer tipo de 'uso', puede, para decirlo sin mayor precisión, ser considerado convencional, en el sentido de que por lo menos es posible explicitarlo mediante la fórmula realizativa, cosa que no ocurre con el último. Así, podemos decir 'te prometo que' o 'te advierto que', pero no podemos decir 'te persuado que' o 'te alarmo que'". (PA, 103, 147-148; cf. también, 105, $149 ; 106,141 ; 120,166)$.

(d) ¿Cuáles son las características de los enunciados que describen los disintos tipos de actos? Es interesante citar los ejemplos que ofrece Austin (particularmente, por el uso que Searle hace de ellos en relación con la segunda de sus críticas). En lo que respecta a la distinción entre actos fáticos y actos réticos, Austin da "... las siguientes parejas de expresiones...

'Él dijo "Estaré en casa" [acto fático]; 'Él dijo que estaría en casa' [acto rético];

"Él dijo "Vete" [acto fático]; 'Él dijo que me fuera' [acto rético];

‘Él dijo "¿Está en Oxford o en Cambridge?" " [acto fá. tico]; 'Él preguntó si estaba en Oxford o en Cambridge' [acto rético].” (JA, 95, 139).

En cuanto a la distinción entre actos locucionarios, actos ilocucionarios y actos perlocucionarios, Austin presenta el siguiente ejemplo: 
"Ejemplo 1:

Acto (A) o Locución.

Él me dijo "Déselo a ella", significando [queriendo decir] con 'déselo' déselo y refiriéndose con 'ella' a ella. Acto (B) o Ilocución.

Él me aconsejó (ordenó, instó a, etc.) que se lo diera a ella.

Acto (C) o Perlocución.

C.a) Él me persuadió que se lo diera a ella.

C.b) Él hizo (consiguió) que se lo diera a ella..." (PA, 101-102, 146).

(e) ¿Cuál es el contenido de las nociones de significado y fuerza? Austin admite la posibilidad de usar la palabra 'significado' con un sentido tal que abarque las dos nociones que desea distinguir: "... podemos hablar de 'significado' para referimos a la fuerza ilocucionaria... Pero deseo distinguir fuerza y significado, entendiendo por este último 'sentido' y 'referencia', tal como ha llegado a ser esencial distinguir sentido y referencia dentro del significado. Además, aquí tenemos un ejemplo de los diferentes usos de la expresión 'usos del lenguaje', o 'uso de una oración', etc. 'Uso' es una palabra demasiado amplia, incurablemente am. bigua, tal como lo es la palabra 'significado', que muchos no toman hoy con seriedad. Pero 'uso', su reemplazante, no está en una posición mucho mejor. Podemos poner totalmente en claro cuál ha sido el 'uso de una oración' en una ocasión particular, en el sentido de acto locucionario, sin tocar siquiera el problema de su uso en el sentido de acto ilocucionario". (PA, 100, 144-145). Por su parte, 'significado' es tomada por Austin, “. . . en la acepción filosófica preferida del término, esto es, con cierto sentido y cierta referencia". (PA, 94, 139). Aunque señala, "Estamos autorizados a sospechar que la teoría del 'significado' como equivalente a 'sentido' y 'referencia', ha de requerir algún desbroce y reformulación sobre la base de la distinción en- 
tre actos locucionarios e ilocucionarios... Reconozco que no he hecho bastante, he aceptado el viejo par de conceptos 'sentido' y 'referencia' bajo el influjo de las opiniones corrientes". (PA, 143, 196). Quizá esté relacionado con tal desbroce y reformulación algo dicho al pasar al referirse a los actos réticos: "El sentido y la referncia (nombrar y referir), son en sí actos accesorios realizados al realizar el acto 'rético'. Así, podemos decir 'Usé "banco" con el significado de..., y también "Cuando dije "él" me estaba refiriendo a ...", $(\mathrm{PA}, 97,141)$.

5. Pondré fin aquí a este collage de temas y de textos extraídos de la doctrina de Austin. Todos los rubros elegidos poseen interés intrínseco y, sólo con diferencias de grado, todos ellos tienen especial relvancia para la discusión que sigue. Es posible que en el acto de seleccionar los textos y en la manera de agruparlos pueda haber influido un afán interpretativo del que no soy consciente. Pero creo - aún cuando tal fuera el caso- que la muestra es representativa y que a través de ella es posible percibir las motivaciones, los planteos y los objetivos teóricos que Austin tuvo, realizó y persiguió al desarrollar su doctrina de los actos lingüísticos. Queda en claro, por lo demás, que no pienso que tal doctrina - considerada globalmente o en aspectos particulares- esté exenta de objeciones. Aunque esta es, sin duda, una cuestión distinta. Lo que me interesa es realzar aquí el sentido de la misma y contar con un telón de fondo sobre el cual se proyectarán con mayor nitidez.las críticas específicas formuladas por Searle y mi discusión acerca del valor téorico de tales críticas.

\section{"Algunos actos locucionarios son actos ilocucionarios"}

6. El primer rasgo que Searle encuentra criticable en la distinción que Austin traza entre actos locucionarios y actos ilocucionarios es que no sea "...totalmente general, en el sentido de acotar clases excluyentes de actos". (LIA, 407). 
Y no es totalmente general porque "....algunos actos locucionarios son actos ilocucionarios". (LIA, 407). Esto ocurre, a su vez, porque “. . . para algunas oraciones, el significado, en el sentido de Austin, determina (al menos), una fuerza ilocucionaria de la emisión de la oración". (LIA, 407).

Los casos paradigmáticos en los que se produciría esta situación especial serían aquellos en los que se efectúa un uso realizativo de verbos ilocucionarios, es decir, casos del tipo 'Te prometo que mañana lo haré', como distintos de casos del tipo 'Mañana lo haré'. La manera como Searle describe el funcionamiento de ambos tipos de casos es, en suma, la siguiente. En el ejemplo 'Mañana lo haré', la emisión seria (esto es, no efectuada al enseñar un idioma, o al recitar una poesía, o al practicar pronunciación, o al actuar en una representación teatral, etc.) y literal (esto es, no metafórica, o sarcástica, etc.) de la oración, puede tener distintas fuerzas ilocucionarias: puede tratarse de una promesa, de una predicción, de una advertencia, etc. Pero cualquiera sea el caso, la oración 'Mañana lo haré' tiene un sólo significado literal. Es más, si conserva ese significado literal y la identidad de referencia, distintas emisiones de dicha oración pueden considerarse casos concretos de un mismo tipo locucionario. "Pero esas mismas emisiones, con el mismo sentido y referencia, podrían ser cualquier acto ilocucionario". (LIA, 406-407); en otras palabras “. . .emisiones de la oración que fueran casos concretos diferentes del mismo tipo locucionario podrían ser casos concretos de diferentes tipos ilocucionarios". (LIA, 407). En el ejemplo 'Te prometo que mañana lo haré, en cambio, las cosas son distintas. Si la emisión de la oración es seria, literal y exitosa (en el sentido de que "... el acto es exitoso, esto es, que las condiciones de emisión exitosa se cumplen". (LIA, 407 , nota 4)), el significado de la oración determina al menos un acto ilocucionario de cierto tipo. En el ejemplo dado, el acto ilocucionario de prometer. "El significado de 
la oración - aclara Searle- determina una fuerza ilocucionaria de sus emisiones, de modo tal que las emisiones serias de ella con ese sentido literal tendrán esa fuerza particular". (LIA, 407).

Searle no indaga los detalles de esta determinación de una fuerza ilocucionaria por parte del significado de la oración. Señala el caso y extrae ciertas consecuencias que, según su opinión, se siguen de él. Al respecto observa: "La descripción del acto como un acto locucionario felizmente realizado, puesto que involucra el significado de la oración, es ya la descripción de un acto ilocucionario, dado que mediante ese significado se determina un acto ilocucionario particular. Ellos son uno y el mismo acto... [porque] cuando cierta fuerza es parte del significado, cuando el significado determina una fuerza particular, no hay dos sentidos distintos sino dos rótulos para el mismo acto... al abstraer el significado de la oración se abstraerá necesariamente una fuerza ilocucionaria, toda vez que la fuerza está incluída en ese significado". (LIA, 497-408). Es importante advertir que al decir esto Searle no pone en tela de juicio la validez de la distinción trazada entre el concepto acto locucionario y el concepto acto ilocucionario. Es más, reconoce expresamente que se trata de dos conceptos distintos. Pero alega que aunque se trate de conceptos diferentes, ellos denotan - como mostraría, a su juicio, el ejemplo en cuestión - clases (parcialmente) coextensivas (overlapping classes). En general, "... en casos tales como el uso realizativo de verbos ilocucionarios el intento de abstraer el significado locucionario de la fuerza ilocucionaria sería como abstraer hombres no casados de solteros". (LIA, 408). En base a todo esto Searle considera probada la primera de sus críticas, a saber, "... que la distinción locucionarioilocucionario no es totalmente general porque algunos actos locucionarios son actos ilocucionarios". (LIA, 408).

Podría argüirse que este planteo no afecta en definitiva la distinción trazada por Austin toda vez que siempre es 
posible distinguir entre el acto locucionario (por ejemplo, decir 'Te prometo que mañana lo haré') y realizar con éxi. to el acto ilocucionario correspondiente, pues este último exige el cumplimiento de un conjunto de condiciones que no se requieren para la realización del primero. Pero Searle rechaza esta posible objeción alegando que lo que en realidad pone de manifiesto es otra distinción, mucho menos interesante que la distinción entre actos locucionarios y actos ilocucionarios, a saber, la distinción entre intentar la realización de un acto ilocucionario y realizar con éxito dicho acto ilocucionario. $Y$ agrega: "...como las condiciones de éxito para la realización del acto son -excepto para las condiciones generales válidas para cualquier tipo de comunicación lingüística - una función del significado de la oración, entonces emitir seriamente la oración con su sentido literal será, al menos, proponerse realizar un acto ilocucionario [determinado, v. gr., prometer, ordenar, etc.]. Y la única distinción que quedará respecto de tales oraciones será la distinción entre esa parte de intentar la realización de un acto ilocucionario que consiste en emitir la oración seriamente y con su sentido literal, y tener éxito realmente en la realización de un acto ilocucionario". (LIA, 409).

7. La crítica de Searle se apoya, como se ve, en un aparato teórico respetable. La cuestión está en determinar si además de ser respetable dicho aparato teórico es aceptable. $\mathrm{Y}$ esto es, precisamente, lo que me propongo discutir.

A modo de comentario preliminar formularé dos breves observaciones. La primera es que Searle no ha sido el único filósofo que advirtió la eventual dificultad que plantea el uso realizativo de verbos ilocucionarios respecto de la distinción locucionario-ilocucionario. Él mismo cita a Cohen

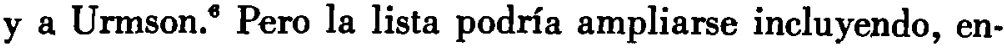

6 L. J. Cohen, "Does Illocutionary Forces Exist?", Philosophical Quarterly, 14 (1964), pp. 118-137; J. O. Urmson, "J. L. Austin", Encyclopedia of Philosophy, compilador: P. Edwards, New York, Random House, 1968, vol. I, pp. 211-215. Searle señala, además, que Austin era consciente de esta dificultad cf. LIA, p. 408 , nota 6 ). 
tre otros, a Strawson y a Hare. ' Todos ellos comparten, en términos generales, la misma preocupación, pero se diferencian en el modo de plantear la dificultad que da origen a tal preocupación y, sobre todo, en las implicaciones teóricas que extraen de tal planteo. El caso extremo lo protagonizan Cohen y el propio Searle. Cohen utiliza el ejemplo del uso realizativo de verbos ilocucionarios como uno de los argumentos que, a su criterio, permiten probar que no existe tal cosa como la fuerza ilocucionaria. Searle -como acabamos de ver - apunta en la dirección contraria; el caso mostraría que algunos actos locucionarios son actos ilocucionarios. Tratándose de una discusión entre filósofos quizá esta situación no deba asombramos. Pero el caso es lo suficientemente sintemático como para llamar la atención y merecer una indagación detallada. Dados los fines que persigo en este trabajo, sólo me limito a puntualizar el hecho. La segunda observación se refiere al reconocimiento de la dificultad por parte del propio Austin. Como hemos visto (cf. nota 6 in fine), Searle indica que Austin era consciente de la misma, aunque no cita texto alguno. Pero me parece que al finalizar la Conferencia X Austin roza el tema. Allí señala: "Los verbos que hemos clasificado... como nombres de actos ilocucionarios parecen hallarse muy cerca de los verbos realizativos explícitos, porque podemos decir 'Te advierto que' y 'Te ordeno' como realizativos explícitos. $\mathrm{Y}$ advertir y ordenar son actos ilocucionarios". (PA, 130, 177). Más adelante agrega: "Pero entonces, ¿cuál es la relación entrè los realizativos y estos actos ilocucionarios? Pareciera que cuando tenemos un realizativo explícito tenemos también un acto ilocucionario". (PA, 131, 178). Y creo que luego de esta reflexión se está obviamente a un paso de plantear la pregunta por la relación entre locuciones e ilocuciones, entre significado y fuerza.

7 P. F. Strawson, "Intention and Convention in Speech Acts", Philosophical Review, 75 (1964), pp. 434460; R. M. Hare, "Austin's distinction between Locutionary and Illocutionary Acts", en R. M. Hare, Practical Inferences, Londres, Macmillan, 1971- pp. 100-104. 
Estas son las dos observaciones preliminares prometidas. Al margen de su posible interés, entiendo que autorizan a pensar que es lícito sostener que el uso realizativo de verbos ilocucionarios plantea una dificultad real e interesante. $Y$ creo, además, que no es descaminado reconocer que tal interés emana del hecho de que dicho uso constituye un caso limite en cuanto a la aplicación de las nociones de acto locucionario y de acto ilocucionario $\mathrm{y}$, paralelamente, de las nociones de significado y fuerza.

Entrando ahora de lleno en la discusión del problema, considero que la línea argumentativa más directa a seguir contra la crítica de Searle consiste en cuestionar la validez de la afirmación de que en el uso realizativo de verbos ilocucionarios el acto locucionario y el acto ilocucionario son uno y el mismo acto. Al respecto puede argumentarse que dado el planteo de Austin -que Searle sigue en sus lineamientos generales al plantear su crítica- ${ }^{8}$ resulta en realidad imposible que se los pueda concebir de tal manera, salvo desvirtuando - cosa que me parece que hace Searleciertas concepciones básicas de aquél.

Supongamos que A y B conversan y que en determinado momento oímos que $A$ dice a $B$ : 'Te prometo que mañana lo haré'. Si nos proponemos describir el acto locucionario que $A$ realizó (o que pudo haber realizado), una respuesta sintética sería "A dijo "Te prometo que mañana lo haré"'. Esta respuesta supone ciertas condiciones para la descripción del acto que corresponden a sus aspectos fonético, fático y rétorico. Si nos proponemos describir, a continuación, el acto ilocucionario que A realizó (o que pudo haber realizado) y si, además, tenemos presente la crítica de Searle, surge el siguiente interrogante: ison las mismas las condiciones para la descripción del acto ilocucionario, que las condicio-

8 Este punto es importante en lo que sigue. La regla implícita que Searle observa, correctamente, a lo largo de sus dos críticas es que ellas se pueden formular dentro del propio planteo de Austin y haciendo uso de su propio aparato teórico-conceptual. Esto fue señalado ya en el parágrafo 2. Está claro, pues, que todo intento de replicar a Searle debe seguir la misma regla, es decir, debe ser hecho desde el propio planteo de Austin. 
nes que hemos tomado en cuenta para describir el acto locucionario realizado por A? Porque si tales condiciones fueran las mismas, entonces la descripción de los dos actos que atribuimos a A sería la misma y, en consecuencia, sería correcto afirmar que no cabría hablar de dos actos distintos sino de uno y el mismo acto.

Considero que la respuesta a este interrogante debe ser negativa. Bien vistas las cosas, tiene que admitirse que para poder producir una descripción adecuada del acto ilocucionario realizado por A se deben tomar en cuenta un conjunto de condiciones que no están incluídas en la descripción del acto locucionario que A realizó. Ejemplos de esas condiciones son, entre otros, que lo que A "prometió" hacer mañana no sea, digamos, una acción que llevaría normalmente a cabo en el curso de su relación con $B$, o que tal acción se encuentre dentro de su ámbito normal de posibilidades, o que A tenga efectivamente la intención de llevar a cabo dicha acción, o que $B$ prefiera que $A$ realice la acción "prometida" a que no la realice, etc. Determinar cuál o cuáles de estas condiciones pueden ser consideradas parte del significado de 'Te prometo que...' es, sin duda, una importante cuestión teórica a dilucidar. Pero si se admite el sentido que Austin otorga a la palabra 'significado' (cosa que, repito, hace Searle) resulta claro que no puede sostenerse que ellas sean parte o formen parte del significado de 'Te prometo que mañana lo haré'. En realidad, esas (y otras condiciones) constituyen un marco de referencia en base al cual podemos llegar a afirmar, por ejemplo, que $\mathrm{A}$ prometió y que su locución 'Te prometo que mañana lo haré' tiene la fuerza de una promesa. Con otras palabras, la pregunta acerca del acto ilocucionario realizado por A puede promover la respuesta canónica ' $A l$ decir "Te prometo que mañana lo haré", A prometió' (realizó el acto de prometer). Esta respuesta no es vacua ni carente de sentido ${ }^{\circ}$ sino que es perfectamente pertinente. $Y$ su pertinencia se

o Adviértase que alguna de estas dos alternativas se siguen necesariamente del planteo de Searle. 
deriva del hecho de que existe un conjunto de condiciones que hacen a la posibilidad misma de describir el acto locucionario realizado por $\mathbf{A}$ como 'teniendo la fuerza de una promesa' y, dado el contenido de la descripción correspondiente, como un acto (ilocucionario) distinto del acto locucionario realizado. Es cierto que él uso del realizativo explícito 'Te prometo...' cumple un papel especial que, por lo demás, el mismo Austin se ha encargado de analizar: en condiciones adecuadas hace explícita la fuerza de lo que se dice. Pero, sólo en condiciones adecuadas. Es cierto, también, que corrientemente decir 'Te prometo...' importa llevar a cabo el acto de prometer. Pero este hecho se relaciona con la normalidad que rige nuestras transacciones lingüisticas y no tiene que ver con el problema conceptual en discusión. En suma, puede concluirse en términos generales que aún en el caso del uso realizativo de verbos ilocucionarios tiene perfecto sentido y es correcto además sostener que las condiciones que tenemos que tomar en cuenta para describir a un acto como acto ilocucionario son distintas de la condiciones relativas a la descripción de un acto como acto locucionario. Si tal es el caso, las descripciones correspondientes a cada tipo de acto serán, pues, distintas, como lo son, en definitiva, los actos.

Pueden imaginarse varias réplicas a esta línea argumentativa. Podría argumentarse, por ejemplo, que lo argüido supone interpretar la distinción locucionario-ilocucionario con un criterio distinto al que Searle emplea. Searle puntualiza claramente que para que tal distinción sea relevante tiene que ser general y que para que sea general tiene qu acotar dos clases mutuamente excluyentes de actos. Todavía puntualiza expresamente que esta es la manera en que la distinción debe ser interpretada (cf. LIA, 413). Lo que prueba Searle - podría agregarse - es que en el caso del uso realizativo de verbos ilocucionarios la distinción locucionario-ilocucionario no es general en el sentido indicado; y este punto no ha sido rebatido de acuerdo a su enfoque específico. No 
hay inconveniente, en principio, en conceder que ello es así. Pero con la salvedad de que, precisamente, una de las cosas que cuestiona la línea argumentativa propuesta es que la distinción locucionario-ilocucionario tenga que ser y deba ser interpretada como sugiere Searle. Adviértase que no sostengo que el criterio empleado por Searle sea criticable en sí mismo, pues si se decide hablar estríctamente en términos de clases de actos, una distinción entre clases diferentes de actos será general si cumple con el requerimiento de acortar clases mutuamente excluyentes. ${ }^{10}$ Lo que sostengo es que interpreta la distinción que traza Austin entre actos locucionarios, actos ilocucionarios, etc., en términos de clases (de actos) es, lisa y llanamente, incorrecto y, en consecuencia, distorsionante de su pensamiento. Esta afirmación tiene sustento en PA. Austin rechaza explícitamente, por ejemplo, la idea de que los actos fonéticos puedan ser considerados una subclase de los actos fáticos (cf. $\$ 4,(5)$ (a) in fine). También declara que la pregunta general es cómo están relacionadas entre sí las distintas descripciones de 'lo que hago' (cf. $\S 4$, (1) in fine). Estas referencias, entre otras posibles, ${ }^{11}$ dan pie para afirmar que las distinciones que propone Austin entre diferentes tipos de actos, las "abstracciones" de que habla, deben ser consideradas como una propuesta de descripciones alternativas del acto lingüístico total 0 , si se prefiere, de descripciones de aspectos, niveles o dimensiones distintas de dicho acto. ${ }^{12}$ Por cierto que el

10 Un ejemplo grueso. Si me propongo distinguir entre actos moralmente buenos y actos moralmente malos y sugiero una caracterización de ambos tipos de actos que sirve, naturalmente, para fundar la distinción entre una y otra clase, está claro que tal caracterización no será adecuada (no será general) si de ella resulta que, en algunos casos, un acto que es moralmente bueno (según la caracterización) es moralmente malo (idem), y viceversa.

11 Cf., por ejemplo, los conocidos textos que se encuentran en PA, pp. 98, 143 y $96,139-140$.

12 Otro ejemplo grueso. El planteo de Austin respecto de las "abstraccio. nes" formulables a partir de un mismo acto parece seguir un modelo del siguiente tipo: el acto por el cual $A$ ha privado de la vida a $B$ puede ser descripto como 'A mató a $B$ ', 'A asesinó a B', 'A cometió homicidio en la persona de B', 'A cometió un delito de naturaleza penal que tuvo como sujeto a B', etc. 
tema de la descripción de acciones (en general) y el de la descripción de actos lingüísticos (en particular) constituyen un vasto campo para una investigación sistemática sólo intentada en parte, pero esta circunstancia es ajena a la posibilidad de decidir acerca de la manera cómo Austin encaró la cuestión $y$, consiguientemente, acerca de la evaluación negativa que merece la interpretación que Searle ha dado a ella.

Podría argumentarse, desde otro ángulo, que pese a todo, la crítica de Searle sigue en pie pues, al margen del interés que pueda poseer la línea argumentativa propuesta, se pasa por alto una importante salvedad que él ha hecho, a saber, que no debe confundirse la distinción locucionario-ilocucionario con la distinción menos interesante que se puede trazar entre intentar la realización de un acto ilocucionario y realizarlo exitosamente. Y - puede agregarse- la línea argumentativa que se sugiere incurre típicamente en tal confusión. Considero, sin embargo, que tal no es el caso. Es más, considero que es Searle quien ha pasado por alto una distinción crucial, esto es, la distinción entre (a) intentar la realización de un acto ilocucionario y/o realizarlo exitosamente y (b) distinguir las condiciones que hacen posible la descripción de tal intento $\mathrm{y} / \mathrm{o}$ realización, es decir, que hacen posible en general la descripción de un acto lingüistico como acto ilocucionario, distinto, por cierto, del acto locucionario correspondiente. Estas son creo yo, cosas diferentes que es necesario separar. Y está claro que al argumento presentado contra Searle presupone admitir que ello es así.

También podría argüirse que la línea argumentativa presentada pasa por alto, sugestivamente, una de sus afirmaciones más reiteradas: que la fuerza puede ser parte del significado. Y podría sostenerse que en realidad éste es un punto básico a rebatir pues lo que el caso del uso realizativo de verbos ilocucionarios pone dramáticamente sobre el tapete es, precisamente, esa posibilidad: que la fuerza sea parte 
del significado. Puede contestarse a esto, por una parte, que si la argumentación propuesta es viable se sigue de ella que aún en el caso del uso realizativo de verbos ilocucionarios siempre hay un sentido legítimo de 'fuerza' y de 'significado' según el cual la primera es distinta de la segunda, con lo cual la tesis de que la fuerza puede ser parte del significado queda fuera de la cuestión. Pero, sin perjuicio de ello, pueden formularse, por otra parte, al menos tres observaciones. La primera es que dado que Searle usa 'sig. nificado' con el sentido poco preciso que Austin atribuye a la palabra y dado que admite - visto el planteo básico de Austin - que la fuerza es distinta del significado (cf. $\$ 4$, (5) (e) ), para que la afirmación 'la fuerza es parte del sifnificado' (y similares) tenga un contenido teórico relevante se impone una aclaración, por mínima que sea, acerca de qué quiere decir que la fuerza es parte del significado. $Y$ tal aclaración está ausente de la trama argumentativa desarrollada por Searle. La segunda observación es que cuando se analiza con cuidado el peso teórico efectivo que tiene dentro de esa trama la afirmación 'la fuerza es parte del significado', se advierte una curiosa ubicuidad que raya en la circularidad. El argumento que formula Searle sigue, en términos generales, el siguiente camino: la distinción locucionario-ilocucionario no es general porque en algunos casos los actos locucionarios son actos ilocucionarios; en tales casos, el acto locucionario y el acto ilocucionario son uno y el mismo acto; a su vez, son uno y el mismo acto cuando el significado de la oración determina unívocamente una fuerza ilocucionaria (al menos); y ello ocurre cuando la fuerza es parte del significado; pero esta última situación se dá cuando, en tales casos, dadas ciertas condiciones restrictivas impuestas a la emisión de la oración, ${ }^{18}$ no

\footnotetext{
13 Es pertinente preguntarse hasta qué punto esas condiciones restrictivas, v. gr., emisión seria, empleo literal de las expresiones lingüísticas, emisión exitosa, no implican, en parte, condicionar ad hac la presentación de la dificultad que preocupa a Searle. Creo que algo puede decirse en este sentido, aunque no desarrollaré aquí el argumento.
} 
hay manera de abstraer el acto locucionario sin abstraer un acto ilocucionario; y “... abstraer el significado de la expresión necesariamente abstraerá una fuerza ilocucionaria, toda vez que la fuerza está incluída en el significado". (LIA, 407-408, las cursivas son mías). Si esta breve esquematización del argumento de Searle es correcta, puede advertirse fácilmente que la afirmación 'la fuerza es parte del significado' juega en él un curioso papel ubicuo y, además, que pareciera servir de puntal de una argumentación que tiene que probar, precisamente, que en ciertos casos la fuerza es parte del significado. La tercera observación es, simplemente, que el criterio que da Searle para poder determinar cuándo la fuerza es parte del significado - a saber, cuando nos vemos imposibilitados de abstraer el acto locucionario sin abstraer un acto ilocucionario- no resulta suficiente. Ello es así porque, precisamente, lo que está en cuestión en si siempre puede ser posible abstraer un acto locucionario sin abstraer, necesariamente, un acto ilocucionario. Este punto es lo que he intentado probar más arriba.

8. Hasta aquí los comentarios referentes a la primera de las críticas de Searle. Pero antes de concluir esta parte deseo formular un breve comentario. Más arriba he señalado que el uso realizativo de verbos ilocucionarios plantea un inteersante caso límite en relación con las nociones de acto locucionario y de acto ilocucionario $\mathrm{y}$, paralelamente, con las nociones de significado y fuerza. He intentado mostrar luego que la primera de las críticas que formula Searle no es aceptable. (Y lo mismo intentaré hacer respecto de la segunda de sus críticas). No hay cabida, pues, en este trabajo para llevar a cabo un enfoque constructivo del problema de fondo. Sin embargo, como mera indicación formulada al pasar, sugiero que una manera plausible de encararlo debe, al menos, (a) hacerse cargo del hecho, no siempre advertido, de que en algún sentido de 'significado' (sentido a precisar, por supuesto), el significado de una oración emitida en un contexto conversacional siempre implica una li- 
mitación de sus fuerzas ilocucionarias posibles, (b) advertir que esa circunstancia puede permitir la construcción de un continuo en el cual el uso realizativo de verbos ilocucionarios sería un caso límite, (c) establecer, dentro del caso límite, las diferencias importantes que pueden mostrar ejemplos distintos (compárese, por ejemplo, la aplicabilidad de los pares locucionario-ilocucionario y significado-fuerza en 'Te prometo que...', 'Te advierto que...', 'Te pido discul. pas') y (d) llevar a cabo este programa mínimo dentro del esquema interpretativo general que he opuesto al de Searle.

"Todos los actos locucionarios son actos ilocucionarios"

9. La primera de las críticas de Searle tiene, por así decir, una alcance limitado y un objetivo en parte preparatorio, pretende valer para un tipo de casos respecto de los cuales resultaría válido afirmar que el acto locucionario es un acto ilocucionario. La segunda de sus críticas, en cambio, tiene un alcance general: cualquiera sea el caso, "...todos los miembros de la clase de actos locucionarios (realizados en la emisión de oraciones completas) son miembros de la clase de actos ilocucionarios, porque todo acto rético $\mathrm{y}$, en consecuencia, todo acto locucionario, es un acto ilocucionario". (LIA, 413).

El punto de partida de la argumentación de Searle es también distinto al anterior. No son ya "casos especiales" los que crean la dificultad sino el contenido de ciertos textos de Austin. Si se analizan los ejemplos que da Austin de actos locucionarios y de actos ilocucionarios (cf. $\$ 4,(5)$ (d) in fine) se advertirá que en los enunciados correspon. dientes usa discurso directo para los primeros y discurso indirecto para los segundos. ("Acto (A) o Locución. Él dijo "Déselo a ella", significando [queriendo decir] con 'déselo' déselo y refiriéndose con 'ella' a ella. Acto (B) o Ilocución. Él me aconsejó (ordenó, instó a, etc.) que se lo diera a ella..."). A su vez, al ejemplificar la distinción entre actos fáticos y actos réticos Austin usa, respectivamen. 
te, discurso directo y discurso indirecto (cf. $\S 4,(5)$ (d)). ("Él dijo "Estaré en tu casa", [acto fático]; "Él dijo que estaría en casa' [acto rético] ...").

Podría pensarse que se trata de una mera gaffe de Austin. Pero Searle, con razón, no considera siquiera una interpretación tan superficial. Se inclina a pensar, en cambio, que tal coincidencia es prevista por Austin y admite que no tendría para su teoría visos de inconsistencia. En el caso específico de la distinción entre actos fáticos y actos réticos, "... dado que el acto locucionario es definido como la emisión de una oración con un sentido y una referencia (significado) entonces ese sentido y esa referencia determinarán un discurso indirecto apropiado para describir el acto locucionario". (LIA, 4I1). Por ejemplo, la forma interrogativa '¿Está en Oxford o en Cambridge?' determina la forma indirecta ' $E$ ĺl me preguntó si estaba en Oxford o en Cambridge'.

Searle centra su crítica en este punto pues observa que las frases verbales que aparecen en las descripciones de actos réticos contienen verbos ilocucionarios, en el sentido de Austin. Además -y esto es lo fundamental- no podrían dejar de contener verbos de tal tipo. Es cierto que se trata de verbos ilocucionarios muy generales, pero ese carácter no le quita que sean verbos ilocucionarios. "Los verbo que aparecen en los ejemplos que da Austin de descripciones en discurso indirecto de actos réticos son todos verbos ilocucionarios de un tipo muy general; tales verbos tienen con los verbos que aparecen en las descripciones de actos ilocucionarios la relación del género a especie". (LIA, 411). Por ejemplo, la frase verbal que aparece, en el nivel rético, en la forma indirecta que corresponde al modo imperativo (' $\mathrm{He}$ told me to...', 'Él me dijo que...') tiene como especies 'ordenó', 'mandó', 'pidió', 'urgió', 'aconsejó', etc. De todo esto Searle concluye que “...al caracterizar los actos réticos, Austin los ha caracterizado, sin advertirlo, como actos ilocucionarios". (LIA, 412). Además, no pudo evitar hacerlo 
así, pues “. . . no hay manera de dar una descripción en discurso indirecto de un acto rético (realizado en la emisión de una oración completa) que no sea una descripción de un acto ilocucionario". (LIA, 412).

El diagnóstico que da Searle de esta curiosa situación contiene varios puntos interesantes. Por un lado observa que "...la distinción locucionario-ilocucionario está diseñada para dar cuenta de aquellos actos en los que el significado de la oración es, por asi decir, neutral respecto de la fuerza". (LIA, 412). Pero - señala- en rigor de verdad nunca se da tal caso. "Toda oración tiene algún potencial de fuerza ilocucionaria, aunque sólo sea de un tipo muy general, insertado en su significado. Por ejemplo, las más primitivas de las vetustas categorías gramaticales de oraciones indicativas, interrogativas e imperativas contienen ya determinadores de fuerza ilocucionaria". (LIA, 412). Y esto es, para Searle, fundamental, porque por tal razón resulta utópico proponerse especificar un acto locucionario (más específicamente, un acto rético) sin especificar un acto ilocucionario. Es más dada esta situación, puede afirmarse que en la distinción trazada por Austin entre actos locucionarios y actos ilocucionarios, no hay actos réticos como cosa distinta de actos ilocucionarios (cf. LIA, 412). Por otro lado, Searle vuelve a señalar, también en este caso, que los conceptos acto locucionario y acto ilacucionario son conceptos diferentes, "...tal como son diferentes los conceptos terrier y perro. Pero la diferencia conceptual no es suficiente para establecer una distinción entre clases separadas de actos, porque asi como todo terrier es un perro, todo acto locucionario es un acto ilocucionario". (LIA, 413).

10. La crítica de Searle se basa, también en este caso, en un aparato teórico respetable. Pero cabe preguntarse, nuevamente, por su aceptabilidad. $Y$ esto es lo que me propongo analizar.

Creo conveniente destacar, en primer lugar, que también en su segunda crítica Searle apunta a una problemática 
válida. No se trata ya -como en el caso de-la primera de sus críticas- de sopesar la viabilidad de la distinción locucionario-ilocucionario frente a lo que constituye un caso límite. Se trata, más bien, de cuestionar ahora los límites mismos de tal distinción, precisamente en su nivel más delicado, esto es, en el de la relación entre acto rético y acto ilocucionario. Resulta interesante señalar, en segundo lugar, que el propio Austin no parece ser totalmente ajeno a las dificultades que pueden surgir, justamente en un punto muy cercano al que Searle toma como tema inicial de su crítica. Luego de dar la lista ya mencionada de ejemplos de actos fáticos y actos réticos, Austin observa: ". . .el acto 'rético' es el que describimos, en el caso de las aserciones, diciendo 'Él dijo que el gato estaba sobre el felpudo', 'Él dijo que iría', 'Él dijo que me tenía que ir' (sus palabras fueron 'Tienes que irte'). Este es el llamado 'discurso indirecto'. Si el sentido y la referencia no han sido entendidos con claridad, entonces toda la expresión o parte de ella, tiene que ir entre comillas. Así, podría decir, 'Él dijo que fuera a ver al "ministro", pero no dijo a qué ministro'... Sin embargo, no siempre podemos usar 'dijo que' con comodidad: si se ha usado el modo imperativo diríamos 'indicó', 'aconsejó' o frases equivalentes. Compárese 'dijo que' con 'presentó sus disculpas', 'dió las gracias', etc." (PA, 96.97, 140-141). Este último párrafo posee una relevancia especia] pues una parte importante de la cuestión pareciera consistir en llegar a determinar la naturaleza de esa "comodidad" (o "incomodidad") en el uso de 'dijo que' de la que nos habla Austin.

Este comentario nos permite entrar directamente en el planteo de Searle. Conviene récordar que Searle acepta el uso de discurso indirecto en la descripción de actos ilocucionarios. También concede que Austin no peca de inconsistencia al describir en discurso indirecto tanto actos ilocucionarios como actos réticos. Lo que le parece criticable, obviamente, es que no se advierte un hecho que, según su 
criterio, es importante: que en la descripción en discurso indirecto de actos réticos aparezcan formas verbales ilocucionarias y que, consiguientemente, no se consideren las consecuencias teóricas que se seguirían de ello. Por tal razón, es pertinente preguntarse, ¿cuál es, realmente, el peso teórico de tal hecho? Y, en el supuesto caso de que pueda ofrecerse a esta pregunta una respuesta interesante, cabe inquirir entonces acerca de la plausibilidad de las consecuencias que Searle extrae. Los argumentos que-ofrezco a continuación intentarán mostrar que, al menos de la manera como Searle desarrolla la cuestión, no se puede dar a la pregunta más que respuestas vacuas. $Y$, si tal es el caso, consideraré probado que las consecuencias que extrae Searle carecen de fundamento adecuado. La técnica argumentativa que emplearé posee un carácter dialéctico que, espero, no produzca mayores confusiones.

Confrontados con el "descubrimiento" de que las descripciones en discurso indirecto de actos réticos incluyen formas verbales ilocucionarias (v. gr., 'preguntó', 'me dijo que' ('he told me to')), puede argüirse en contra de Searle que sus implicaciones teóricas son más que relativas. Si la cuestión se reduce a la mera aparición en el nivel rético de formas verbales del tipo 'Me preguntó si...', por ejemplo, se podría intentar la reformulación de todas las descripciones en discurso indirecto de actos réticos eliminando dichas formas verbales y usando, únicamente, la fórmula general 'dijo que'. Por ejemplo:

'Él dijo "Estaré en casa", [acto fático]; 'Él dijo que estaría en casa [acto rético];

'Él dijo "Vete", [acto fático]; 'Él dijo que me fuera [acto rético];

'Él dijo “¿Está en Oxford o en Cambridge?"' [acto fático]; 'Él dijo (que) si estaba en Oxford o en Cambridge' [acto rético].

En los tres casos aparece la forma verbal 'dijo que'. Y 
si bien se percibe cierta "incomodidad" en el tercero de los casos, tal elemento no puede tomarse prima facie como un criterio decisivo para rechazar el punto central del argumento, esto es, la posibilidad de reformular todas las descripciones en discurso indirecto de actos réticos empleando la forma verbal 'decir que' en su 'acepción plena y normal" (cf. $\S 4,(2)$ ). De.tal manera quedarían eliminadas las formas verbales ilocucionarias.

Este argumento podría ser rebatido por un defensor de Searle. Podría decirse, por ejemplo, que no es más que una manera -quizá ingenua- de pasar por alto el problema de fondo, pues no se trata - tal como supone el argumen. to-- de discutir la mera aparición y la eventual elimina. ción de formas verbales ilocucionarias. Se trata, en realidad, de algo distinto, a saber, de algo que podría caracterizarse como la ilocucionariedad propia de la forma. verbal con la que describimos en discurso indirecto los actos réticos. La tesis es que toda descripción de tal tipo incluye necesariamente verbos ilocucionarios que poseen, por ser tales, fuerza ilocucionaria. Y - podría agregarse- el argumento anterior invierte los términos de este planteo; no se trata de que porque aparecen en esas descripciones verbos ilocucionarios se afirme dicha tesis, sino de que la tesis se hace cargo de un hecho que se manifiesta necesariamente en la aparición, a nivel rético, de tales verbos.

Considero importante analizar esta eventual réplica. Ella reitera lo afirmado por Searle. Pero lo hace ahora eliminando cierta ambigüedad implícita en su planteo. En este contexto resulta claro que dicho planteo no surge de la mera constatación de que a nivel rético aparecen formas verbales ilocucionarias, sino que el mismo se basa en la idea de que esas formas verbales tienen, toda vez que aparecen en ese nivel, fuerza ilocucionaria. Y resulta obvio, creo, que de ser ese el caso Searle tiene que ofrecer una fundamentación adecuada a su tesis, que trascienda la referencia a ejemplos. Mostrar esto es el valor que posee el argumento eventualmente "ingenuo" formulado más arriba. 
Cabe preguntarse entonces, ¿qué fundamentación firme ofrece Searle en favor de su tesis? Y lo cierto es que no resulta fácil encontrar una respuesta convincente a esta pregunta. Podría apelarse a la imposibilidad -alegada por Searle-de abstraer un acto rético sin abstraer un acto ilocucionario. Pero esta respuesta no sería aceptable. En realidad - de ser ese el caso- la eventual "respuesta" registraría una consecuencia de la tesis y nunca un fundamento de ella. ¿Qué otra cosa podría ofrecerse como fundamento de la tesis? Creo yo que el único recurso disponible consiste en recurrir a la observación, sin duda interesante, de que los modos gramaticales clásicos poseen potencial ilocucionario, pues tal observación pareciera sugerir, al menos, lo siguiente: que lo que a nivel fático se describe, por ejemplo, como una oración e nmodo interrogatvo, se debe describir a nivel rético por medio de una oración que contiene una frase verbal canónica como, por ejemplo, 'Me preguntó si... Y Y tal frase verbal tiene furza ilocucionaria.

Pero, ¿qué interpretación precisa puede darse a esta observación? ¿Cuál es el peso teórico efectivo de la tesis que afirma que los modos gramaticales poseen "potencial ilo. cucionario"? Ambas preguntas son pertinentes, pues argüiré que su peso teórico es más promesa que realidad y que, en definitiva, esta tesis tiene un contenido prácticamente nulo que no alcanza para sustentar la tesis de fondo a la que Searle debe dar fundamento.

Adviértase, en primer lugar, que así como la forma gramatical de una oración no coincide necesariamente con su forma lógica (esto es, al menos, lo que desde Bradley en adelante nos han inculcado varios filósofos ilustres), tam. poco el modo gramatical de una oración determina unívo. camente una fuerza ilocucionaria específica. Es un lugar común que, por ejemplo, una oración en modo indicativo

\footnotetext{
14 Cabe agregar que, de cualquier manera, el planteto de que no es posible abstraer un acto rético sin abstraer un acto ilocucionario puede ser atacado siguiendo una línea argumentativa similar a la ofrecida en relación con la primera crítica.
} 
puede ser usada para informar, describir, afirmar un hecho, etc., pero también para instar a hacer algo, ordenar algo, indicar cierto comportamiento a seguir, etc. $Y$ no es difícil imaginar el uso, en cada caso, de verbos ilocucionarios adecuados. Observaciones similares pueden hacerse respecto del modo imperativo y del modo interrogativo. En suma, no se ve muy bien, desde este punto, qué puede pretender implicar la tesis referente al "potencial ilocucionario" de los modos gramaticales clásicos.

Adviértase, en segundo lugar, que si tal como sostiene Searle, la descripción en modo indirecto de actos réticos contiene necesariamente verbos ilocucionarios, tampoco se ve por qué razón no podríamos llegar a producir pares de descripciones del siguiente tipo:

“Él dijo “¿Porqué no se lo dá a ella?” [acto fático]; 'Él me instó $a$ (pidió, aconsejó, urgió, etc.) que se lo diera a ella' [acto rético].

Este ejemplo debe ser rechazado por Searle. Y debe serlo porque - según se podría alegar- no se adecúa a su planteo. Lo que él sostiene es que en el nivel rético los verbos que aparecen en las descripciones correspondientes poseen un carácter ilocucionario muy general y, además, que son géneros que acotan a nivel de la descripción de los actos ilocucionarios familias de verbos ilocucionarios que son especies de dichos géneros. $\mathrm{Y}$, obviamente, el ejemplo dado no cumple con esas condiciones. Creo que corresponde conceder el punto, pero con la salvedad de que a esta altura de la argumentación la tesis acerca del "potncial ilocucionario" de los modos gramaticales clásicos y, consiguientemente, la tesis general que sustenta la segunda de las críticas formuladas por Searle enfrentan un dilema de dudosa solución. $\mathrm{O}$ bien los verbos que aparecen a nivel rético son verbos ilocucionarios en un sentido pleno y estricto, en cuyo caso - dado que el modo gramatical no determina necesariamente una específica fuerza ilocucionaria- pueden pro- 
ducirse válidamente ejemplos como el anterior, con lo cual se disuelve la tesis de Searle acerca de la relación géneroespecie entre los verbos "ilocucionarios" que aparecen a nivel rético y los que aparecen a nivel ilocucionario, o bien el carácter ilocucionario de las formas verbales que se dan a nivel rético - carácter que Searle enfatiza- exige ser provisto de un contenido que no es proporcionado por la argumentación de Searle. Y ello debe ser así, so pena de que el argumento "ingenuo" con que inició estas consideraciones críticas tenga un valor refutativo efectivo.

Creo que a esta altura de la discusión hay elementos suficientes para avalar la observación de que la tesis básica de Searle no tiene un fundamento firme o, si se prefiere, que la fundamentación genérica que ofrece resulta teóricamente vacua. Si tal es el caso, es dudoso aceptar la consecuencia que Searle extrae en su segunda crítica, esto es, que los actos réticos son actos ilocucionarios. Por cierto que queda en pie el hecho de que, tal como observa Searle, Austin incluye en la descripción de los actos réticos verbos que en el nivel ilocucionario son verbos ilocucionarios. También queda en pie la necesidad de ofrecer un diagnóstico adecuado de tal hecho, que por lo demás pareciera haber sido advertido por el propio Austin. Pero estas circunstancias no afectan ni el sentido ni el valor de las observaciones formuladas. Tampoco son óbice para rechazar la segunda de las críticas de Searle por carecer de fundamento adecuado.

Como complemento de lo anterior me interesa formular dos observaciones adicionales. La primera se relaciona con la tesis sustentada por Searle de que la distinción trazada entre actos locucionarios y actos ilocucionarios "...está diseñada para dar cuenta de aquellos casos en los que el significado de la oración es, por así decirlo, neutral respecto de la fuerza", (LIA, 412) y que, en realidad, "... toda oración tiene algún potencial ilocucionario". (LIA 412), podría decirse que nada se encuentra más cercano al planteo 
de Austin que esta última idea. Recuérdese, por ejemplo, que para Austin realizar un acto locucionario es, en general, eo ipso, realizar un acto ilocucionario. Y esto implica, entre otras cosas, que toda oración tiene "potencial ilocucionario". Searle descarta este tipo de consideraciones y produce una interpretación deformada del planteo de Austin. Creo que al trazar la distinción locucionario-ilocucionario, Austin no pensó en casos en los que la oración pudiera ser neutral respecto de la fuerza. Y ello es así, sencillamente, porque tales casos no parecen existir en las transacciones lingüísticas cotidianas. Lo que parece haber pensado Austin al proponer la distinción locucionario-ilocucionario es -como he argumentado en relación con la primera de las críticas de Searle- ofrecer un esquema teórico que abarque descripciones alternativas del acto lingüístico, de cuya aplicación resulta la distinción entre lo que la oración emitida en cierta situación conversacional significa y la fuerza que dicha oración posee en esa situación. Pero esto es, por cierto, diferente a lo que sugiere Searle.

Es interesante señalar, en segundo término, que también en este caso Searle admite que el concepto locucionario es distinto al concepto ilocucionario, aunque la manera como acotan clases es diferente a la descripta en el contexto de la primera crítica. Antes se sostuvo que no se podía abstraer el acto locucionario del acto ilocucionario, tal como no se pueden abstraer no casados de solteros. Ahora, la imposibilidad de abstraer un acto del otro es equiparada a la imposibilidad de abstraer un perro de un terrier. La relación es de especie a género (de subclase a clase). Ya he criticado el enfoque que subyace a esta terminología. Lo que me interesa apuntar aquí es que existe una obvia diferencia entre la formulación relativa a la primera crítica y la formulación relativa a la segunda. Si tal diferencia se tomara en serio podría argumentarse que altera nada menos que el significado del verbo 'ser' en la oración 'Los actos 
locucionarios son actos ilocucionarios'. En el contexto de la primera crítica la oración equivaldría a decir 'Actos locucionarios $=$ actos ilocucionarios', mientras que en el contexto de la segunda crítica la oración equivale a 'Los actos locucionarios son una subclase de los actos ilocucionarios'. Y quizá tenga importancia destacar esta ambigüedad como un elemento adicional para valorar el peso efectivo de las críticas de Searle.

11. Creo que todo lo dicho hasta aquí es suficiente como para poner en tela de juicio la posibilidad de aceptar la segunda de las críticas que formula Searle. No está claro, en modo alguno, en qué sentido los actos réticos son, o pueden ser, actos ilocucionarios. La tesis de que los modos gramaticales poseen potencial ilocucionario carece de un contenido mínimo aceptable, y la propuesta acerca de la existencia de verbos ilocucionarios genéricos que acotarian clases de especies de verbos ilocucionarios no es susceptible de implementación o resulta directamente irrelevante. EI cuadro es lo suficientemente obscuro como para agregar comentarios adicionales.

\section{Algunas observaciones finales}

12. Al comenzar este trabajo precisé las consecuencias que estaba dispuesto a reconocer a las críticas formuladas a Searle. Advertí que no pensaba que críticas de este tipo pudieran llegar a conmover el esquema teórico desarrollado en Speech Acts. Señalé también que pensaba que, de ser correctas, estas críticas mostrarían que una de las posibles líneas argumentativas que llevan a ese esquema carece de asidero. Y agregué que éste no era, por cierto, un punto mínimo. En ese momento di una razón general acerca de por qué no se trataba de un punto mínimo. Pero considero pertinente concluir el trabajo ofreciendo una razón más es. pecífica. En pocas palabras, se trata de lo siguiente. Como lo muestra la estructura y el contenido del artículo de 
Searle, la segunda de las críticas que formula a Austin crea, o pretende crear, la motivación necesaria para justificar la introducción de los actos proposicionales (a decir verdad, una curiosa especie, de "actos"). La "reducción" del nivel rético al nivel ilocucionario abre el espacio teórico necesario para la ubicación de este nuevo tipo de actos y del aparato conceptual que trae aparejado. Todo esto surge de manera expresa en el artículo de Searle. La que no aparece $\tan$ claramente es la finalidad de la primera crítica. Es correcto, por cierto, considerarla, dado su carácter restringido, como un primer paso en la argumentación que luego se profundiza con la segunda crítica. Pero, sin perjuicio de aceptar esto, no me parece del todo descaminado señalar que en la primera de las críticas hay atisbos de lo que luego va a ser la estrategia general diseñada por Searle para tratar a la fuerza como parte del significado, estrategia en la que son piezas claves el ya mencionado "principio de expresibilidad", la especificación de las condiciones necesarias y suficientes para realizar determinado acto lingüístico (v. gr., prometer), la "extracción" de reglas para el uso del indicador de fuerza ilocucionaria, a partir de tal conjunto de condiciones, etc.

Cuando se las ubica en estos contextos, se advierte que las objeciones que he formulado a Searle poseen un peso que puede llegar a ser importante. Probar que en el punto de partida de un enfoque teórico (el de Searle), que pretende ser una superación de otro enfoque teórico (el de Austin), no se ha dado una valoración ni un análisis crítico satisfactorio a un tema tan decisivo como es la distinción austiniana entre actos locucionarios y actos ilocucionarios plantea, al menos, la necesidad de una revisión. $Y$ esa revisión se impone, no por meras delicadezas exegéticas sino para intentar ofrecer un fundamento más claro a la teorización acerca de los actos lingüísticos. Naturalmente que esto presupone admitir que las objeciones formuladas contra Searle son adecuadas. Que lo sean -acepto- es algo que puede ser, a su vez, tema de discusión. 
1." In "Austin on Locutionary and Illocutionary Acts", Sections I and II, John Searle argues (1) that Austin's distinction between locutionary and illocutionary acts "cannot be completely general in the sense of marking off two mutually exclusive classes of acts, because for some sentences, at least, meaning, in Austin's sense, detrmines (at least one) illocutionary force of the utterance of the sentence" (p. 407), and (2) that "all members of the class of locutionary acts (performed in the utterance of a complete sentence) are members of the class of illocutionary acts, because every rhetic act, and hence, every locutionary act, is an illocutionary act" (p. 413).

2. This paper discusses both theses and purports to show that they lack the conclusiveness that Searle is ready to foster upon them. An analysis of Searle's arguments brings to light several difficulties, such as a doubtful reading of Austin's basic tenets and an appeal to distinctions which are not clearly stated and/or not sufficiently grounded.

3. The paper begins with a review of some of Austin's doctrines ( $\$ 4$ and 5). Searle's thesis (1) and (2) are summarized and discused in $\S \S 6-8$ and $\S \S 9-11$, respectively. Some concluding remarks are made in $\S 12$.

4-5. A collage of Austin's main tenets is presented almost vorbatim within a framework which highlights (1) the nature and aim of Austin's doctrine, (2) the disctintion between locutionary, illocutionary and perlocutionary acts, (3) the interest in the illocutionary act, (4) some consequences which follow from (3), and (5) some difficulties in Austin's doctrine, such as (a) the nature of speech acts and the relationship between them, (b) the criteria for the identification of speech acts, (c) the conventionality of locutionary and illocutionary acts, (d) the features of the reports of spech acts, and (e) meaning and force.

6. Searle's first thesis asserts that the distinction between locu. tionary and illocutionary acts is not completely general because some locutionary acts are illocutionary acts. And this is so because,

* Paragraph's numbers correspond to those of the paper. 
for some sentences, meaning determines at least one illocutionary force of the utterance of the sentence. A paradigmatic example of such sentences is found in the performative use of illocutionary verbs (e.g., 'I hereby promise that I am going to do it', as opposed to 'I am going to do it'). In those cases, "the meaning of the sentence determines an illocutionary force of its utterances in such a way that serious utterances of it with that literal meaning will have that particular force. The description of the act as a happily performed locutionary act since it involves the meaning of the sentence, is already a description of the illocutionary act, since a particular illocutionary act is determined by that meaning. They are one and the same act". (p. 407). Searle does not explain how it is that a particular illocutionary act is determined by the meaning of a certain sentence. He just points this out and proceds to elaborate without further comments. Moreover, Searle admits that the concept of a locutionary act is a different concept from the concept of an illocutionary act (same admission in the second thesis), but he notices that although different they denote overlapping classes. On the other hand, Searle does not accept that the distinction between locutionary and illocutionary acts could be read as a distinction between th simple meaningful sentence and the succesfully performed complete illocutionary act. He does so on the ground that this way out reduces the locutionary-illocutionary disttinction to the less interesting distinction between trying and suceeding in performing an illocutionary act.

7. In relation to Searle's first thesis it is observed (a) that the problem posed by the performative use of illocutionary verbs has been pointd out already by Cohen, Urmson, Strawson, Hare, etc., and that it is interesting to notice that they differ in the statement of the problem and in the assessment of its consequences, and (b) that Austin seems to be aware of the problem (cf. HTD, pp. 130-1). It is argued, further, that Searle's contention, i.e., that in the performative use of illocutionary verbs, the locutionary act and the illocutionary act are one and the same act, is untenable, this "consequence" does not follow from Austin's position. In fact, an adequate description of an illocutionary act must always take into account a set of conditions which are not included (cannot be included) in the adequate description of the corresponding locutionary act (e.g., in the case of promising, the normal course of action, the possibility of performing the action, the having of an adequate intention, the preference for having the action performed, etc.). This set of conditions is a framework to which the very possibility of asserting, for instance, 'I hereby promise...', 'S pro- 
mised...', etc., is referred. It follows that the description of an ilocutionary act differs, in the above mentioned sense, from the description of a locutionary act; and then, that both acts are different. It also follows that the canonical form 'In saying "I hereby promise...", he was promising' is not void or senseless (a consequence of Searle's thesis). It could be replied that this approach (a) does not merit the fact that Searle's argument is based among other things, on the possibility of distinguishing mutually exclusive classes of acts, (b) is an example of mixing up the locutionaryillocutionary distinction with the distinction between trying and succeeding in performing an illocutionary act, and (c) does not take into account that the force of an utterance can be part of its meaning. But it is answered, (a) that Searle's talk about classes of acts is a flagrant violation of Austin's points about the relation. ship between different kinds of linguistic acts (cf. HTD, p. 96, p. 122), (b) that Searle's distinction between trying and suceeding presupposes a more basic one: the distinction between different sets of conditions that make possible the description of an act and (c) that Searle's references to the force as part of the meaning are utterly unclear and, is some sense, circular.

8. Finally, some comments are made about some difficulties related to the performative use of illocutionary verbs and the possibi. lity of approaching them on different grounds.

9. Searle's second thesis states that all members of the class of locutionary acts are members of the class of illocutionary acts, because every rhetic act is an illocutionary act. Searle notices that in reporting rhetic acts Austin makes use of indirect speech and that in the corresponding reports he uses illocutionary verbs (e.g., 'say', 'ask', 'tell to') of a very general kind, which -according to Searle- stand in relation to the verbs used in the reports of illocutionary acts as genus to species. Searle thinks that the locutionary-illocutionary distinction is designed to account for cases where the meaning of the sentence is force-neutral. But, "every sentence has some illocutionary potential, if only of a very general broad kind built into its meaning... even the most primitive of the old-fashioned grammatical categories of indicative, interrogative, and imperative sentences already contains determinants of illocu. tionary force. For this reason, there is no specification of a locution. ary act performed in the utterance of a complete sentence which will not determine the specification of an illocutionary act... There are no rhetic acts as opposed to illocutionary acts at all." (p. 412). 
10. The problem that underlies Searle's thesis is a real one. Moreover, Austin himself seems to be aware of it (cf. HTD, pp. 96-7). But Searle's arguments are very far from being acceptable. In fact, an analysis of them shows that his basic tenets are void, i.e., they lack theoretical weight. A first step towards proving this point consists in sorting out Searle's initial gambit by reducing every report of a rhetic act into the canonical form 'He said that...'. It can be argued of course that this is a naive movement; that it is not a question of what verbs are used but of the illocutionary character which is shown, necessarily, by the verbal forms included in the reports of rhetic acts. If so, Searle owes us an adequate explanation of this necessity. A natural step to make in this direction is to resort to the illocutionary potential of the grammatical categories. But this step does not take us very far. First, it is clear that the grammatical form does not determine univocally a certain illocutionary force. Second, the relationship genus-species, pointed out by Searle, is easily dispeled by way of examples. Moreover, it is not correct to assert, as Searle does, that the locutionary-illocutionary distinction is designed by Austin to account for cases where the meaning of the sentence is force-neutral, but that in fact, no sentence is force-neutral. It is quite clear that Austin never conceived of sentences, actually used, as force-neutral, and it is difficult to see, then, how the distinction between locutionary and illocutionary acts could have been drawn on this basis.

11. It is concluded that Searle's second thesis is not sufficiently grounded and presupposes a doubtfull reading of Austin's position.

12. Some brief remarks are made about the relationship holding between both theses and some basic doctrines of Speech Acts. The second thesis is clearly related to the introduction of propositional acts. The first one seems to be related to Searle's strategy towards treating force as part of the meaning, the principle of expressibility, the statement of necessary and sufficient conditions for the performance of speech acts, etc. If valid, the criticism of both theses cancel a possible grounding for Searle's general strategy. But this criticism does not affect the strategy itself. 